«Presque au-delà de la mode».

La semiotica di genere nel linguaggio di Prada

"Presque au-delà de la mode ". La sémiotique du genre dans le langage de Prada

\title{
Francesca Sensini
}

\section{(2) OpenEdition}

\section{Journals}

Edizione digitale

URL: http://journals.openedition.org/cei/1179

DOI: $10.4000 /$ cei. 1179

ISSN: 2260-779X

\section{Editore}

UGA Éditions/Université Grenoble Alpes

\section{Edizione cartacea}

Data di pubblicazione: 30 juin 2013

Paginazione: 135-155

ISBN: 978-2-84310-245-5

ISSN: 1770-9571

Notizia bibliografica digitale

Francesca Sensini, ««Presque au-delà de la mode».

La semiotica di genere nel linguaggio di Prada», Cahiers d'études italiennes [Online], 16 | 2013, online dal

15 décembre 2014, consultato il 27 mars 2021. URL: http://journals.openedition.org/cei/1179 ; DOI: https://doi.org/10.4000/cei.1179 


\title{
«PRESQUE AU-DELÀ DE LA MODE» . LA SEMIOTICA DI GENERE NEL LINGUAGGIO DI PRADA
}

\author{
Francesca Sensini \\ Université de Nice
}

La moda parla una lingua di immagini che ha la prerogativa di rappresentare emblematicamente il contesto sociale nel quale essa è prodotta, diventandone insieme lo specchio e la proiezione ideale. L'iconografia di moda assolve, in effetti, una funzione socio-culturale che travalica il messaggio commerciale strictu sensu. Lo scopo ultimo rimane la vendita di un prodotto ma il processo che vi conduce offre ai professionisti del settore notevoli possibilità di espressione e sperimentazione. In particolare, è per mezzo delle immagini pubblicitarie che la moda riesce a oltrepassare le sue finalità immediate, per veicolare presso il pubblico non soltanto nuovi modelli consumistici, ma anche inedite elaborazioni dell'io. I suoi messaggi di natura identitaria, nondimeno, si iscrivono in un discorso che risulta fondamentalmente paradossale: se da un lato, in effetti, la moda chiama in causa l'affermazione dell'identità, dall'altro essa promuove il conformismo, proponendo in modo normativo un ventaglio ristretto e prêt-à-porter di modelli. Tuttavia, è interessante rilevare che, per quanto riguarda le rappresentazioni di genere, la pubblicità di moda può interpretare una coscienza più complessa e profonda di quella di altri media. Non di rado, in effetti, essa riflette un pannello di generi più ampio e diversificato, in contrasto con la rappresentazione dei sessi veicolata da altri tipi di pubblicità e dalla televisione in generale, in cui si constata ancora la tendenza a svalutare l'esperienza e le competenze delle donne, in favore di immagini incentrate sulla bellezza plastica ed esaltanti stereotipi legati ai ruoli tradizionali.

I. Prada Book, Prefazione di Patrizio Bertelli e Miuccia Prada, Milano, Progetto Prada Arte, 2009, p. II (in francese nel libro). 
Collocandosi al vertice del paesaggio socio-economico, le industrie del lusso - come Prada, che è oggetto della nostra analisi - impiegano grandi mezzi finanziari e creativi per proporre immagini pubblicitarie - o parapubblicitarie, come video, cortometraggi, lookbook, ecc. - affascinanti e cariche di significati emozionali, capaci di trasmettere messaggi incisivi. Tuttavia, nonostante i metodi sofisticati, i mezzi finanziari e le energie creative impiegate massivamente nella realizzazione di un'iconografia estremamente ricercata e originale, la gamma identitaria proposta dai marchi del lusso appare generalemente assai ristretta: uomini e donne giovani, rimandi a un elevato status sociale e a una sessualità, quale che sia, tradizionale o post-genere, spesso esacerbata. Per capire le ragioni di una tale tendenza, così come le trasgressioni, seppur rare, a questo modello, sarebbe importante determinare le ideologie soggiacenti alle immagini di moda e ricostruirne la genesi; ma questa operazione risulta molto difficile, poiché esse sono oggetti culturali derivati da un processo creativo complesso, al quale concorrono diverse figure professionali specializzate (art director, fotografi, stylist, ecc.), ognuna portatrice di significati culturali diversi. In effetti, se possiamo agevolmente identificare il proprietario di queste immagini, ovvero la marca di cui esse mostrano il logo, non è facile determinarne l'autore.

Alla luce di queste considerazioni, cercheremo di affrontare il problema delle rappresentazioni di genere nel linguaggio di Prada basandoci anche sulla bibliografia ufficiale della maison - comunicati stampa, libri e interviste - che ci permette di definire con più precisione, risalendo direttamente alla fonte delle informazioni, le iniziative e le intenzioni creative di Prada e le strategie di comunicazione che queste tendono a dissimulare.

\section{Il caso Prada}

Nel campo del lusso, Prada si impegna nell'adottare una strategia paragonabile a quella dei «Lifestyle Brands»" per distinguersi efficacemente nell'omogeinizzazione caratterizzante le immagini associate al lusso. Poiché le creazioni di moda attengono alla sfera dell'attualità immediata e dell'effimero, Prada ha scelto di contaminare il suo linguaggio con quello dell'arte, fenomeno culturale aspirante a comunicare messaggi permanenti. Nel caso

2. Secondo la definizione di John Grant nel suo saggio The New Marketing Manifesto, Cheshire, Texere, 2000 (prima edizione 1999), un «Lifestyle Brand» — o «Idea Brand» — è un marchio che si prefigge di esprimere i valori dei consumatori; valori che superano una concezione puramente materialistica della vita e veicolano concetti filosofici, etici, politici. 
specifico, la maison si è impadronita delle posizioni avanguardiste dell'arte contemporanea. Profondamente ancorata nella modernità, questa forma d'arte ambisce ad anticipare le tendenze del presente e a rifletterne le movenze, pur mirando a veicolare un messaggio capace di impressionare, non rararamente critico, e durevole. Superando - e confondendo - le frontiere tra strategie di marketing, autopromozione e mecenatismo, l'azienda ha finito per diventare la committente di un'arte che sembra demistificare le sue pratiche di lavoro e il sistema di significati che essa rappresenta ${ }^{3}$.

Accanto a queste forme innovative di autopromozione, Prada sviluppa altresì immagini pubblicitarie di tipo tradizionale, diffuse dalla stampa specializzata, nelle riviste autorizzate e - benché questo resti ancora piuttosto raro e limitato a certi prodotti soltanto - nelle grandi città e alla televisione ${ }^{4}$. La qualità e la ricerca artistica restano gli elementi distintivi di queste pubblicità rispetto a quelle di altri marchi del lusso, a dimostrazione del fatto che l'azienda ambisce a un tipo di comunicazione che generalmente richiede allo spettatore uno sguardo 'attivo'. Possiamo citare a titolo esemplificativo le foto realizzate da Glen Luchford nel 1997 e nel I998, ispirate ai film noir e impostate come frammenti di una narrazione in fieri. È interessante notare come, in luogo di presentare delle immagini suscettibili di ridurre la percezione di distanza tra l'oggetto osservato e l'osservatore, per far scattare così un processo di identificazione, la marca sembra privilegiare un'iconografia aliena, addirittura alienante: le donne e gli uomini sono idealizzati, mancano riferimenti chiari a uno stile di vita glamour — i personaggi hanno l'aria solitaria e distante - e gli scenari sono talmente vaghi e spogli da parere irreali (immagine I) s. Conseguentemente, nessuna identicazione immediata appare possibile;

3. È il caso di opere come Prada Toilet e Prada Death Camp realizzate dall'artista americano Tom Sachs, rispettivamente nel 1997 e nel 1998, o ancora Prada Marfa creato dai norvegesi Michael Elmgreen e Ingar Dragset, nel 2005. Si tratta di progetti nati spontaneamente: Prada Toilet rappresenta delle toilettes di cartone, ricavate a partire da scatole di scarpe Prada. Sempre le scatole da scarpe sono servite a Sachs per creare il suo controverso Prada Death Camp, modello di un campo di concentramento tedesco che, esposto al Jewish Museum di New York, fu all'origine di forti proteste. Prada Marfa è, invece, un'installazione permanente che rappresenta una boutique Prada, situata a circa $60 \mathrm{~km}$ a nord della città di Marfa in Texas, sulla strada 90. Essa è destinata a degradarsi lentamente e ad integrarsi al paesaggio circostante; per questa ragione, non è previsto nessun lavoro di restauro.

4. Fino a oggi le pubblicità programmate alla televisione riguardano solo i profumi dell'azienda. Dal 2008 Prada realizza anche video, la cui diffusione avviene via internet, sul sito ufficiale della maison e sui siti specializzati. Invece, i cartelloni outdoor interessano tutti i prodotti e i luoghi di affissione sono selezionati secondo criteri quali il prestigio e la visibilità; a volte, in occasione di lavori di rinnovamento, in particolare dei negozi monomarca, o flag shops, si possono vedere enormi cartelloni che avvolgono letteralmente gli edifici.

5. Occorre precisare che le foto delle campagne pubblicitarie di Prada - come avviene ormai quasi universalmente - sono postprodotte al computer. 
al contrario, queste immagini propongono ciò che potremmo definire un' «alter-identificazione», cioè l'identificazione con l'immagine di un Alter Ego, a metà strada tra l'Ego et l'Altro, solitario e isolato nella sua eccezionalità.

Per quanto riguarda gli standard fisici, le pubblicità di Prada, come le sfilate stesse, non vengono meno alla pratica - criticata e nondimeno corrente nel settore della moda a tutti i livelli - di mostrare immagini di corpi gracili, talvolta magri all'eccesso (di donne e, per quanto in misura minore, anche di uomini) (immagini 7-8). Non si può affermare, invece, che le immagini pubblicitarie utilizzate dall'azienda enfatizzino il corpo dei modelli; questi ultimi appaiono spesso 'sezionati' per mettere in evidenza solo i prodotti, i soli veri oggetti del desiderio. Coerentemente con questa impostazione, esse non promuovono paradigmi di bellezza impregnati di politiche sessuali e non realizzano messaggi pubblicitari basati su un modello discriminante, in cui un genere 'domina', per così dire, l'altro, come accade non di rado nelle pubblicità di altre marche del lusso. Per restare nell'ambito del made in Italy, si ricordi, a titolo di esempio, la campagna pubblicitaria di Gucci del 2003, ideata da Tom Ford, improntata alla tendenza del cosiddetto porno-chic, le cui scene esplicite di dominazione maschile e una rappresentazione degradante della donna ne determinarono la censura; o ancora la campagna Dolce \& Gabbana del 2009, assai choccante, con scene chiaramente allusive alla violenza sessuale.

Lasciando da parte tendenza porno-chic, stereotipi della donna-oggetto e 'sguardo maschile' — il male gaze che invade le immagini pubblici$\operatorname{tarie}^{6}$ - Prada punta su androginia e coolness, accompagnati da caratteristiche fisiche non di rado atipiche rispetto agli standard di bellezza mainstream (immagini 2-4). Sulla base di queste considerazioni, ci concentreremo sull'analisi dell'iconografia di moda firmata Prada, al fine di evidenziare i concetti di identità e le rappresentazioni di genere che ne derivano. Inizialmente, prenderemo in considerazione un cortometraggio diretto da Ridley et Jordan Scott in collaborazione con Prada nel 2005. In seguito, analizzeremo un cortometraggio di animazione, al centro della campagna Primavera-Estate 2008, Trembled Blossoms. Per concludere, esamineremo le immagini del lookbook uomo e donna della PrimaveraEstate 20IO.

6. John Berger, Questione di sguardi, Milano, Il Saggiatore, I998, p. I42. 


\section{Thunder - Perfect Mind: un'identità femminile onnicomprensiva}

Presentato in anteprima al Festival del cinema di Berlino il is febbraio 2005, il cortometraggio Thunder - Perfect Mind è stato realizzato da Ridley Scott e da sua figlia Jordan in occasione del lancio del primo profumo Prada, ufficialmente senza nome (detto Amber) ${ }^{7}$. Il titolo è la traduzione dal greco Bronté: nồs téleios, "Tuono, intelletto perfetto", titolo di un poema anonimo in lingua copta appartenente alla collezione di testi gnostici detta di Nag Hammadi e risalente al I secolo d. C. ${ }^{8}$. Questo poema è stato suggerito a Miuccia Prada, la mente creativa della maison, come sottofondo sonoro per il film, da Jordan Scott. Con la sua proposta, la regista ha voluto interpretare il desiderio della creatrice milanese di rappresentare la complessità propria dell'essere donna, secondo quanto si legge nel comunicato stampa diffuso dall'azienda: «Il desiderio di Miuccia Prada era di ritrarre la complessità dell'essere donna, e Jordan lo ha interpretato proponendole una poesia anonima del I secolo, Thunder-Perfect Mind, che esplora gli infiniti aspetti della psiche femminile.»

Nel film, infatti, la modella Daria Derbowy — vestita con creazioni realizzate espressamente per le riprese da Miuccia Prada ${ }^{9}$ — declina la donna in una moltitudine di ruoli: sposa, fanciulla, madre, figlia, amante. Il filo rosso che unisce le trasformazioni di Daria Derbowy è rappresentato dai passi in inglese del poema Bronté: noûs téleios ${ }^{\mathrm{IO}}$, che la modella legge nella metro di Berlino. In funzione di commento alle scene che si giustappongono, i versi sviluppano il tema della natura contraddittoria, addirittura paradossale, di un'identità femminile misteriosa che si autorappresenta:

[...] For I am the first and the last. [...]// I am the wife and the virgin./ I am the mother and the daughter. [...] / I am she whose wedding is great, / and I have not

7. Il cortometraggio è visibile in rete sul sito ufficiale di Prada e su varie piattaforme di video sharing.

8. A proposito di questo testo e del corpus di cui fa parte, Giuliana Lanata precisa che «nell'ignoranza o, per meglio dire, nell'incertezza in cui versiamo sull'origine e sulla storia della collezione di testi copti che va sotto il nome di biblioteca di Nag Hammadi [...], si è discusso se i vari testi fossero redatti in greco fin dall'origine, o fossero a loro volta traduzioni greche di originali composti in altre lingue» (Giuliana Lanata, Io sono la sapienza dei Greci e la conoscenza dei Barbari, lezione e traduzione ancora inedita tenuta nel quadro di un seminario presso l'Università di Pavia e al Paul Getty Center di Los Angeles nel 1996).

9. Il comunicato stampa precisa che: «I costumi sono stati appositamente disegnati da Miuccia Prada, ed il loro styling è stato affidato a Kym Barrett, famosa per successi come Matrix e Romeo \& Juliet. Tom Foden, che ha già lavorato in passato con Ridley e Jordan, era il responsabile di produzione mentre le coreografie sono di Blanca Li."

Io. Non si conosce il nome del traduttore del testo in lingua inglese. Il testo che riportiamo rispetta la divisione in versi e strofe della traduzione di Giuliana Lanata, citata alla nota 8, che peraltro precisa che questa presentazione svolge una funzione puramente pratica, semplificando la lettura del poema rispetto alla scriptio continua adottata dal manoscritto copto. 
taken a husband. [...]// I am the bride/ and the bridegroom [...]// I am the silence that is incomprehensible/ and the idea whose remembrance is frequent./

I am the voice whose sound is manifold [...]// I am senseless and I am wise. [...]// II ... and establish the great ones among the small first creatures. / Come forward to childhood, / and do not despise it/ because it is small and it is little. / And do not turn away greatnesses in some parts from the smallnesses, / for the smallnesses are known / from the greatnesses. [...]// I am the one who is honored, and who is praised [...]//

For I am knowledge/ and ignorance./ I am shame and boldness. / I am shameless; / I am ashamed./ I am strength and I am fear. / I am war/ and peace. [...]// But I, / I am compassionate/ and I am cruel. / Do not hate my obedience/ and do not love my self-control. ${ }^{12}[\ldots] / /$ But I am she who exists in all fears/ and strength in trembling./ I am she who is weak, / and I am well in a pleasant place [...]// I am peace, / [...] And I am an alien/ and a citizen.// I am the substance/ and the one who has no substance. [...]// I am control/ and the uncontrollable./ I am the union and the dissolution. [...]// I am the hearing which is attainable to everyone/ and the speech which cannot be grasped. / I am a mute who does not speak, / and great is my multitude of words.

Il personaggio in questione è la Bronté — «il Tuono», nome di genere femminile in greco - divinità gnostica dalla natura androgina, dotata di un «intelletto perfetto». Le sue affermazioni antinomiche esprimono «l'oscillazione tra immanenza e trascendenza» e sembrano rimandare a una "visione dualistica e opposizionale della realtà» ${ }^{\text {⒊ }}$. Appare chiaro che, rispetto all'estrema complessità di questo testo, l'affermazione riguardante "gli infiniti aspetti della psiche femminile» che il poema illustrerebbe appare quanto meno riduttiva. Resta il fatto che i passi selezionati, debitamente estrapolati dal loro contesto storico-culturale, di cui conservano semplicemente il fascino oscuro e prezioso, sono passibili di una tale interpretazione o, meglio, riduzione. Di conseguenza, la dea Bronté finisce per diventare la donna Prada, dall'identità insieme contraddittoria e capace di armonizzare tutti i contrasti. Senza mettere troppo l'accento sull'ambiguità di genere — «I am the bride and the bridegroom» è il solo verso scelto che faccia riferimento all'androginia di Bronté - il cortometraggio allude al problema del genere come costruzione sociale, mettendo innanzi allo spettatore l'immagine di una donna che incarna ruoli opposti su di un piano culturale ma coesistenti entro lo stesso individuo. Così la donna

II. A partire da questo passo, la successione dei versi del testo originale non è più rispettata. Rinviamo all'articolo della Lanata (cfr. nota 8) per una presentazione dettagliata del poema e per una traduzione filologicamente rigorosa e non di seconda mano, sulla base dell'originale copto.

I2. Questo passo si discosta sensibilmente dalla traduzione proposta dalla Lanata: «Non odiate la mia ubbidienza; / e la mia padronanza di me nella debolezza, / amatela.»

13. Giuliana Lanata, lezione citata. 
viene esaltata per la ricchezza perturbante della sua natura e celebrata per il suo mistero, essenzialmente insolubile.

Thunder-Perfect Mind è un chiaro esempio di realizzazione a metà strada tra due sfere creative - il cinema e la moda - in cui ogni riferimento commerciale sembra secondario. In effetti, il flacone del profumo Prada appare soltanto nell'ultima sequenza del film, quando la voce femminile di sottofondo ripete "for I am the First and the Last». Lidea stessa di presentarlo in anteprima al Festival del cinema di Berlino ne fa implicitamente un'opera artistica. Nondimeno, la ricerca artistica e l'originalità dell'iniziativa si riflettono, in qualche modo, sulle merce da promuovere; investita di un valore semantico aggiunto, quest'ultima diventa il simbolo di una femminilità onnicomprensiva, dalle radici antiche ed estremamente moderna - Daria Derbowy è immersa nella lettura del poema Thunder-Perfect Mind su un anonimo sfondo metropolitano, lontana dai luoghi più celebri di Berlino — culturalmente raffinata e consapevole del suo posto nella società contemporanea.

\section{Trembled Blossoms: modelli post-genere}

Proiettato per pochi intimi il 5 febbraio 2008 nella boutique-epicentro di Broadway ${ }^{\mathrm{I}}$, in occasione della settimana della moda di New York, Trembled Blossoms ${ }^{15}$ è un cortometraggio di animazione della durata di quattro minuti circa, girato da James Lima, prodotto da Max Brun di Hi! Production e animato da Sight Effects Los Angeles ${ }^{16}$. Secondo il comunicato stampa diffuso dall'azienda, l'opera è il punto di arrivo di una serie di progetti concepiti da Miuccia Prada parallelamente alla creazione della collezione donna Primavera-Estate 2008 e realizzati con la collaborazione di designer e artisti internazionali:

L'ambizioso progetto comprende, oltre alla collezione di moda, il design di tessuti, l'allestimento della sala sfilata, il wallpaper per specifici ambienti, set fotografici e adesso un film animato. Trembled Blossoms si inserisce nell'impegno costante di Prada per la creazione sperimentale. [...] Miuccia Prada ha ideato un paesaggio fantastico

I4. Per il concetto di boutique-epicentro voir Prada book, cit., p. 420.

I5. Benché nessun documento ufficiale dell'azienda vi faccia scoperto riferimento, il titolo del cortometraggio sembra rimandare ai versi 9-II del poema di John Keats, Ode to Psyche; si tratta del passo in cui si descrive il luogo in cui Eros et Psiche dormono, uno accanto all'altra: «Saw two fair creatures, couchèd side by side/ In deepest grass, beneath the whisp'ring roof/ Of leaves and trembled blossoms, where there ran/ A brooklet, scarse espied.»

16. Come Thunder-Perfect Mind, anche questo cortometraggio è disponibile sul sito ufficiale dell'azienda e sulle principali piattaforme di video sharing. 
che poteva però funzionare sia come ambientazione che come idea per un tessuto. Durante l'estate Miuccia Prada ha sviluppato, in collaborazione con Michael Rock e Sung Kim di $2 \times 4^{17}$, innumerevoli alternative che sono state catalogate. $2 \times 4$ ha poi coinvolto nel progetto James Jean, giovane illustratore di Los Angeles, e insieme hanno prodotto i prototipi per gli Epicentri, lo spazio delle sfilate e gli speciali tessuti. I disegni a inchiostro - attualmente visibili negli Epicentri Prada a New York e Los Angeles — raffigurano un lussureggiante paesaggio di fiori e ninfe, leggermente scandaloso, che evoca la visione di Miuccia Prada, giustapponendo elementi di Art Nouveau, Liberty, Aubrey Beardsley e Hieronymus Bosch.

Pastiche derivato da riferimenti culturali diversi - dall'estetica finde-siècle all'iconografia visionaria, abitata da inquietanti esseri metamorfici del pittore olandese del xv secolo Hieronymus Bosch — Trembled Blossoms coniuga innovazione e tradizione: la tecnica d'avanguardia detta Motion Capture permette, effettivamente, di catturare i movimenti di esseri umani dotati di sensori riflettenti, in modo paragonabile a quello che facevano i disegnatori dei Walt Disney Studios negli anni TrentaQuaranta, lavorando sulle riprese filmate di attori in movimento. Dopo essere state registrate, le tracce dei movimenti sono impresse informaticamente' sui disegni. Grazie a questo metodo, il ricorso all'animazione digitale non intacca lo spirito e la bellezza dei disegni realizzati manualmente.

Dal punto di vista dei contenuti, Trembled Blossoms mette in scena la nascita di un essere femminile dai tratti fortemente stilizzati, il cui corpo, completamente bianco e polito, rimanda a una condizione di innocenza originaria ed evoca, in qualche modo, una tabula rasa su cui tutto deve ancora essere scritto. Su uno sfondo bianco con rameggi in stile liberty, un colibrì becca il cuore tondo e carnoso di un enorme fiore dalle radici fluttuanti. Mentre il fiore si ripiega inesorabilmente sull'animale, una goccia lucente e densa fuoriesce dalla corolla; come fecondata da questa goccia, una tela bianca - allusione al foglio di carta su cui prende vita il disegno - si solleva e si disgrega per lasciare apparire una donna, metà ninfa, metà cyborg. A partire da questo momento, comincia la sua evoluzione: la sua condizione di essere primordiale si modifica progressivamente attraverso l'accesso alla conoscenza. Quest'ultima è simboleggiata, da una parte, dal frutto, un pomo (identico al pistillo del fiore), che il personaggio raccoglie da un ramo solitario che gli si offre (identico alle

17. $2 \times 4$ è uno studio di design situato a New York. Con AMO, lo studio di consulenti esperti in design dell'azienda OMA (Office of Metropolitan Architecture), diretta dall'architetto olandese Rem Koohlas, e con altre figure professionali specializzate, collabora con Miuccia Prada per la realizzazione di wallpapers, esposizioni, animazioni e media interattivi per le boutique-epicentro di New York, Los Angeles e Tokyo. Una scelta di wallpapers creati da Miuccia Prada e da $2 \times 4$ è stata esposta al Museum of Modern Art di New York, nella sezione consacrata all'architettura e al design, nel corso del 2008. 
radici del fiore); dall'altra, dai vestiti e accessori che progressivamente la ricoprono. Strani insetti variegati abbracciano i suoi piedi nudi per trasformarsi in scarpe col tacco; indossate queste ultime, il personaggio può cominciare l'esplorazione dello spazio circostante. Al suo passaggio, una vegetazione lussureggiante si espande, animando il vuoto; una sorta di ombra-doppio la circonda magicamente con un tessuto colorato che aderisce al suo corpo, mentre la nuova Eva di Prada morde serenamente il suo edenico pomo, gettando attorno a sé uno sguardo meravigliato. Alla fine della sua esplorazione, un dio Pan, inquietante e benevolo ad un tempo, la attende in una radura, seduto su di una specie di trono a forma di corolla; da quest'ultimo sgorga improvvisamente una sorgente, che forma un piccolo stagno. È allora che un enorme serpente si leva in volo da dietro il trono di Pan per gettarsi in acqua e trasformarsi in un pesce multicolore. Senza la minima paura, Eva gli getta l'ultimo morso del suo frutto, provocando un'ulteriore trasformazione: il pesce diventa una borsetta su cui si distingue chiaramente il logo di Prada in lettere dorate. Dopo aver accettato la piccola borsa dalle mani di Pan ed essersi messa a volteggiare gioiosamente sotto una pioggia di petali bianchi, Eva assiste a un'ultima metamorfosi: come una scultura di porcellana, l'immagine di Pan si infrange per lasciare apparire nuovamente il fiore gigantesco della prima sequenza. Tutto ricomincia nuovamente: un uccellino tutto fremente esce fuori dalla borsetta colorata per andare a pungere col suo becco il pistillo succoso da cui colerà la goccia generatrice di Eva. Nel frattempo, Eva alza il telo bianco per nascondersi in attesa di rinascere.

Nel quadro di questa genesi in versione Prada, è interessante notare come il personaggio femminile sia fondamentalmente un essere ibrido, per metà umano, per metà fantastico, in sospeso tra due nature: mentre i suoi movimenti sono quelli di una ballerina, filmata secondo la tecnica Motion Capture, il suo corpo è una creazione derivata dall'elaborazione digitale coniugata col disegno tradizionale. Inoltre, il suo corpo bianco, senza tratti chiaramente definiti, appena sessuato, come il suo viso, sembra essere sospeso in una preadolescenza e situarsi prima delle distinzioni di genere dell'età adulta. Il personaggio maschile, dal canto suo, possiede una natura per lo meno composita: capro favoloso e uomo possente, è destinato a trasformarsi nel fiore primordiale che darà origine all'Eva di Prada. Nel contesto del cortometraggio, questa trasformazione, così come il legame con la generazione, non sembrano irrilevanti per il nostro tema: in effetti, il fiore è un organismo vivente dotato di una struttura riproduttiva in cui coesistono androceo e pistillo, ovvero una parte maschile e una parte femminile. Così il Pan di Trembled Blossoms è una creatura 
ermafrodita; in modo simile alla nuova Eva, riunisce e confonde le categorie di genere.

Sulla base di queste riflessioni, la rappresentazione dei personaggi femminili e maschili in Trembled Blossoms, pur entro la cornice di un favola mitologica che sembra, almeno a un primo livello di lettura, senza sorprese, ci rimanda alla nozione di cyborg introdotta da Donna Haraway nella riflessione femminista postmoderna ${ }^{\mathrm{I} 8}$, nella misura in cui questa rappresentazione sovverte le idee di naturalezza e artificialità. Ciò non riguarda soltanto il corpo e il genere, come abbiamo cercato di evidenziare, ma anche l'opposizione classica tra natura e cultura: l'Eva cyborg di Prada passa dalla natura alla cultura — dalla nudità al corpo vestito - mordendo il frutto della conoscenza, per ritornare poi a uno stato primordiale in attesa di rinascere e ricominciare il suo percorso di formazione. Inoltre, gli abiti e gli accessori che vestono la nudità di Eva non sono altro che trasformazioni culturali di elementi e motivi tratti dal mondo naturale, vegetale e animale. Come Eva e Pan, le scarpe-insetto e la borsetta-pesce sono degli ibridi che mettono in crisi la pretesa opposizione tra natura e cultura. Soggetti al flusso delle metamorfosi - allusione ai cambiamenti continui di cui la moda si nutre - i personaggi e l'Eden di Trembled Blossoms sembrano condurre al rovesciamento dei punti di riferimento legati alle nozioni di genere, demistificando le costruzioni culturali che si nascondono dietro le categorie tradizionali d'interpretazione del mondo.

\section{Lookbook Primavera-Estate 20ı0: l'identità palinsesto}

Concepito e realizzato dallo studio di esperti in design AMO, diretto da Rem Koohlas, il fantasy lookbook Primavera-Estate 2010 di Prada rappresenta uomini e donne visti attraverso l'obiettivo fissurato di una camera cinematografica. Il riferimento alla camera cinematografica rinvia all'idea che le foto siano frammenti di una storia che l'osservatore è in grado di ricostruire con l'immaginazione, in armonia con lo spirito di altre immagini Prada. Il ricorso all'obiettivo rotto, dal canto suo, esaspera il carattere irreale e alieno delle immagini di uomini e donne, già caratteristico dell'iconografia del marchio. Infatti, per mezzo di questo sguardo defor-

18. Si veda, in particolare, Donna Haraway, Primate Visions: Gender, Race, and Nature in the World of Modern Science, New York, Routledge, 1989, Simians, Cyborgs and Women: The Reinvention of Nature, London, Free Association Books, I991, e Manifesto Cyborg. Donne, tecnologie e biopolitiche del corpo, a cura di Liana Borghi, introduzione di Rosi Braidotti, Milano, Feltrinelli, r995. 
mante, i corpi dei modelli diventano superfici prive di unità e di contorni stabili, destinate a essere scomposte, ricomposte, ingrandite o rimpicciolite dalla fantasia del fotografo (immagini 5-7). Nello stesso tempo, essi subiscono in un certo qual modo un processo di disumanizzazione: lo sguardo cool, svuotato d'emozione, raramente rivolto all'osservatore, abbinato all'atteggiamento rigido, accentuano la loro funzione di modelli, fino a renderli pure forme, paragonabili a qualunque altra forma inanimata presente sulle foto. In effetti, i corpi umani - o le parti su cui l'obiettivo si focalizza - finiscono per fondersi con le cose: in certe pagine del lookbook si possono vedere immagini di uomini e donne inframmezzate da foto e disegni, alcuni dall'estetica latu sensu futurista, rappresentanti sezioni o frammenti di architettura urbana, come facciate, ponti, metropolitane, palazzi, gallerie (immagini 8-I2). Ogni riferimento esplicito all'appartenenza di genere o a un qualsiasi rapporto gerarchico tra di essi sembra assente: lo spettatore si ritrova di fronte a immagini di creature giovani, a metà strada tra persona e cosa, dalle sagome slanciate, magre, a volte gracili, che galleggiano senza gravità in un universo artificiale; questo stesso carattere artificiale è il segno distintivo che li unisce, collocandoli su uno stesso piano.

Similmente, gli elementi paesaggistici - un paesaggio sempre urbano e in ogni caso marcato dalla civiltà moderna, come per esempio le grandi spiagge disseminate di teli da mare, ombrelloni e gente anonima o gli orizzonti bordati di palmizi e attraversati da aerei - risultano instabili, sfuocati, frammentari. Nella pagina introduttiva del lookbook, i designer di AMO sintetizzano in questi termini i temi che animano lo sfondo delle foto: "AMO glimpses the collection through a fractured cinematographic lens: contorted cityscapes, tropical traces, desolate architecture, illusory projections and palimpsests flicker like movie stills.» È interessante osservare che questa breve descrizione è punteggiata di parole che rimandano a idee di labilità e indeterminazione; in effetti, e gli individui e gli scenari su cui si stagliano appaiono precari e cangianti come immagini chimeriche. Tutto questo sottolinea la nozione di identità precaria e multipla che abbiamo già incontrato nell'esame di altre campagne pubblicitarie. A questi aspetti si aggiunge l'idea di esseri umani in osmosi con l'universo urbano in cui gravitano, fino quasi ad integrarne la materialità: si tratta dunque di un'altra declinazione dell'ibrido firmato Prada; quest'ultima sembrerebbe influenzata dal sogno futurista dell'uomo-macchina - e i riferimenti alle avanguardie storiche sembrano testimoniare in questo senso. 
Frammentati e snaturati nelle loro normali proporzioni, inframmezzati a foto e disegni, attraversati da effetti di luce e macchiati da sbavi di colore che colano dai loro stessi vestiti, le donne e gli uomini del lookbook tradiscono apertamente la loro funzione di palinsesto, come i designer di AMO non mancano di suggerire. Infatti, i loro corpi sono paragonabili a supporti sui quali Prada scrive la sua storia visiva; una storia effimera, destinata a essere cancellata prima dell'inizio di una nuova stagione - e di una nuova campagna pubblicitaria.

Per concludere, gli esempi di campagne pubblicitarie che abbiamo preso in considerazione mostrano come Prada conduca una ricerca multiforme capace di superare il campo della moda per toccare temi più ampi, connessi all'estetica, alla tecnologia, alla comunicazione. Nell'introduzione del libro Prada, che ripercorre la storia della maison milanese fin dai suoi inizi, nel 1913, il presidente e amministratore delegato dell'azienda, Patrizio Bertelli, e Miuccia Prada spiegano le ragioni ideali di questo percorso:

Pour Prada la mode, le luxe et le style constituent un projet qui va au-delà de la production sériale de vêtements, de chaussures et de sacs. Une insatiable curiosité et une observation attentive du monde, de la société et de la culture sont au cour de la créativité et de la modernité de Prada. Sa quête l'a conduite à franchir les limites physiques que représentent les boutiques et les salons d'exposition; elle a provoqué une interaction entre des univers différents et apparemment éloignés, et introduit une nouvelle manière de créer une mode naturelle, presque au-delà de la mode. ${ }^{19}$

Dunque «l'insaziabile curiosità e l'attenta osservazione del mondo» hanno spinto l'azienda ad approfondire il tema dell'identità e del genere nel quadro della società contemporanea, in cui i vestiti non sono più visti unicamente come semplici abbellimenti esteriori, ma finiscono per rivestire la funzione di altrettanti messaggi da decodificare, in stretto rapporto con l'identità della persona che li indossa. Il punto di partenza di questa indagine sembra essere la coscienza che il soggetto contemporaneo, al di là delle differenze di genere, è eccentrico in senso proprio: non può più appoggiarsi su valori assoluti, si discosta da ogni categoria che, definendolo, lo priverebbe della capacità di rinnovarsi senza fine e di essere quindi autenticamente moderno, in accordo con le evoluzioni della società. Ora, quest'aspirazione al cambiamento comporta una perdita di unità, di stabilità, persino di consistenza ontologica. Il lookbook Primavera-Estate 2010 offre un esempio eccellente di questo messaggio: gli uomini e le donne Prada assomigliano a simulacri sfuggenti, inquietanti, quasi ases-

19. Prada Book, cit., p. II. 
suati, talmente in simbiosi con il mondo moderno - rappresentato dalle metropoli - e con la sua ansia di rinnovamento da diventare creazioni avanguardiste, alienate rispetto alla loro stessa natura umana.

Maggiormente incentrato sulla semiotica di genere, Thunder-Perfect Mind sembra demistificare la costruzione socio-culturale dei due sessi e il gioco di ruoli che ne deriva. Nella sua apparente classicità, il film pubblicitario mira a esaltare una complessità che nega l'esistenza di un sé essenziale; questa non può essere colta se non attraverso uno sguardo nuovo, libero dal peso delle vecchie categorie di lettura dei generi e, in particolare, degli stereotipi dell'immagine della donna.

La valorizzazione dei modelli post-genere è al centro di Trembled Blossoms. Il cortometraggio, infatti, prende in considerazione il divario che può esistere tra sesso biologico e identità di genere; lo scopo consiste nel superamento dell'opposizione uomo/donna in favore di un modello, per così dire, ermafrodito e in perenne trasformazione. Il personaggio del cyborg - essere umano, disegno, animazione, ninfa, mostro, avatar - diventa la metafora di questo stesso superamento. È chiaro che siamo di fronte a rappresentazioni di un soggetto multiplo e ambiguo che intacca l'ideologia di genere dominante, imposta non solo dalle agenzie di socializzazione ma anche dai media, di cui, tuttavia, queste immagini pubblicitarie fanno parte. In questo modo, il linguaggio visivo della moda firmato Prada offre il suo contributo al dibattito sull'identità di genere, riuscendo con originalità a esprimere la presa di coscienza dei cambiamenti in atto nella società contemporanea allo stesso titolo degli altri settori della cultura.

\section{Bibliografia}

Baudrillard Jean, Simulacres et simulations, Paris, Galilée, I98I.

Berger John, Questione di sguardi, Milano, Il Saggiatore, 1998.

BetTERTON Rosemary, Looking on: Images of Femininity in the Visual Arts and Media, London, Pandora, 1987.

Bordo Susan, Il peso del corpo, Milano, Feltrinelli, 1997.

Bourdieu Pierre, La distinzione, Bologna, Il Mulino, 1983.

Bovone Laura, Creare comunicazione, Milano, Franco Angeli, 1994.

Bruzzi Stella e Church Gibson Pamela, Fashion Cultures, London, Routledge, 2002.

Cheles Luciano, Immagini di moda e immagini artistiche, in Il vestito e la sua immagine, a cura di Jeanine Guérin Dalle Mese, Provincia di Belluno, 2002, pp. 313-326. 
Crane Diana, Descrivere il mondo, Roma, Carocci, 200I.

-, Fashion and Its Social Agenda, Chicago (IL), University of Chicago Press, 200I.

—, Questioni di moda, Milano, Franco Angeli, 2002.

Cremonesini Valentina e Izzi Carla, La rappresentazione di genere nell'analisi del veicolo pubblicitario, in Il Dubbio, II, 4, 2003, pp. 277-305.

Entwistle Joanne, The Fashioned Body: Fashion, Dress and Modern Social Theory, Cambridge, Polity Press, 2000.

Europe 1910-1939. Quand l'art habillait le vêtement, Paris, Paris Musées, 1997.

Finkelstein Joanne, After a Fashion, Melbourne, Melbourne University Press, 1996.

—, Fashion, New York, New York University Press, 1998.

Fiske John, Understanding Popular Culture, London, Unwin Hyman, 1989. Goffman Erving, Gender Advertisements, New York, Harper and Row, 1976.

Gottdiener Mark, Postmodern Semiotics: Material Culture and the Forms of Postmodern Life, Oxford, Blackwell, 1995.

Grogan Sarah, Body Image, London, Routledge, 1997.

Hall Stuart, Hobson Dorothy, Lowe Andrew e Willis Paul, Culture, Media, Language, London, Hutchinson, 1981.

Haraway Donna, Primate Visions: Gender, Race, and Nature in the World of Modern Science, New York, Routledge, 1989.

-, Simians, Cyborgs and Women: The Reinvention of Nature, London, Free Association Books, I99I

-, Manifesto Cyborg. Donne, tecnologie e biopolitiche del corpo, a cura di Liana Borghi, introduzione di Rosi Braidotti, Milano, Feltrinelli, I995.

Hermes Joke, Reading Women's Magazines, London, Polity Press, 1995.

Jobling Paul, Fashion Spreads: Word and Image in Fashion Photography Since 1980, Oxford, Berg, 1999.

Kawamura Yuniya, La moda, Bologna, Il Mulino, 2006.

McRobbie Angela, In the Culture Society, London, Routledge, I999.

Mort Frank, Boy's Own? Masculinity, Style \& Popular Culture, in Male Order: Unwrapping Masculinity, a cura di Rowena Chapman e Jonathan Rutherford, London, Lawrence \& Wishart, 1988, pp. 193-224.

-, Cultures of Consumption: Masculinities and Social Space in Late Twentieth Century Britain, London, Routledge, 1996.

Mortelmans Dimitri, Socio-Semiotics Analysis of Print Advertisements for Luxury Products, in «Semiotica», I20, I, 2, I998, pp. I8I-206. 
Nixon Sean, Hard Looks: Masculinities, the Visual and Practices of Consumption, London, UCL Press, 1996.

Polhemus Ted e Procter Lynn, Fashion \& Anti-Fashion: An Anthropology of Clothing and Adornment, London, Thames \& Hudson, 1978.

Representation: Cultural Representation \& Signifying Practices, a cura di Stuart Hall, Milton Keynes, Open University Press, 1997.

Rock Michael, Prada Book, Milano, Progetto Prada Arte, 2009.

Tolmach Lakoff Robin e Scherr Raquel L., Face Value: The Politics of Beauty, Boston, Routledge \& Kegan Paul, I984.

Tseelon Efrat, The Masque of Femininity, London, Sage, 1995.

Valentino. Trentanni di magia, Milano, Bompiani, I99I.

Van Zoonen Liesbet, Feminist Media Studies, London, Sage, I994.

Versace Gianni, Vanitas: lo stile dei sensi, a cura di Omar Calabrese, Milano, Leonardo Arte, I99I.

Wolff Naomi, The Beauty Myth, New York, Virago, I99I. 


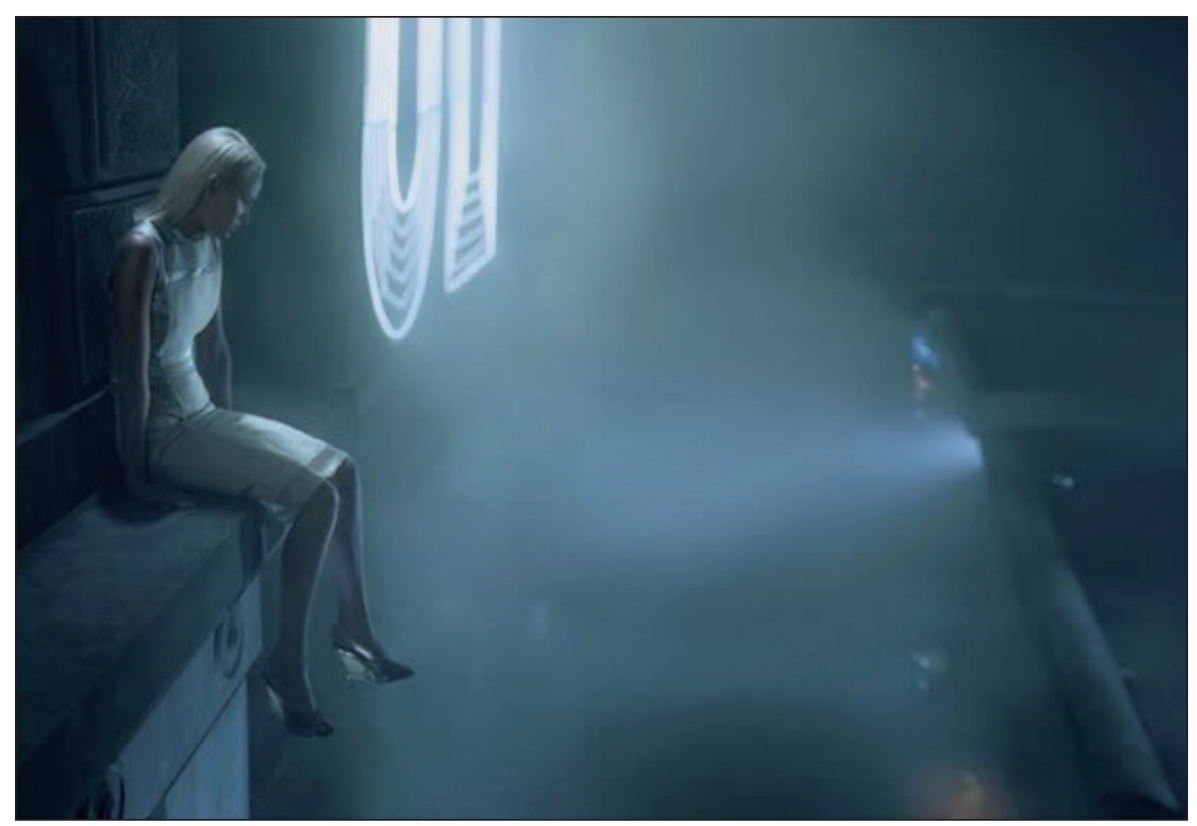

Immagine I. - Prada Primavera-Estate 1998.

Fotografo: Glen Luchford (Art Partner) - Stylist: Alex White.

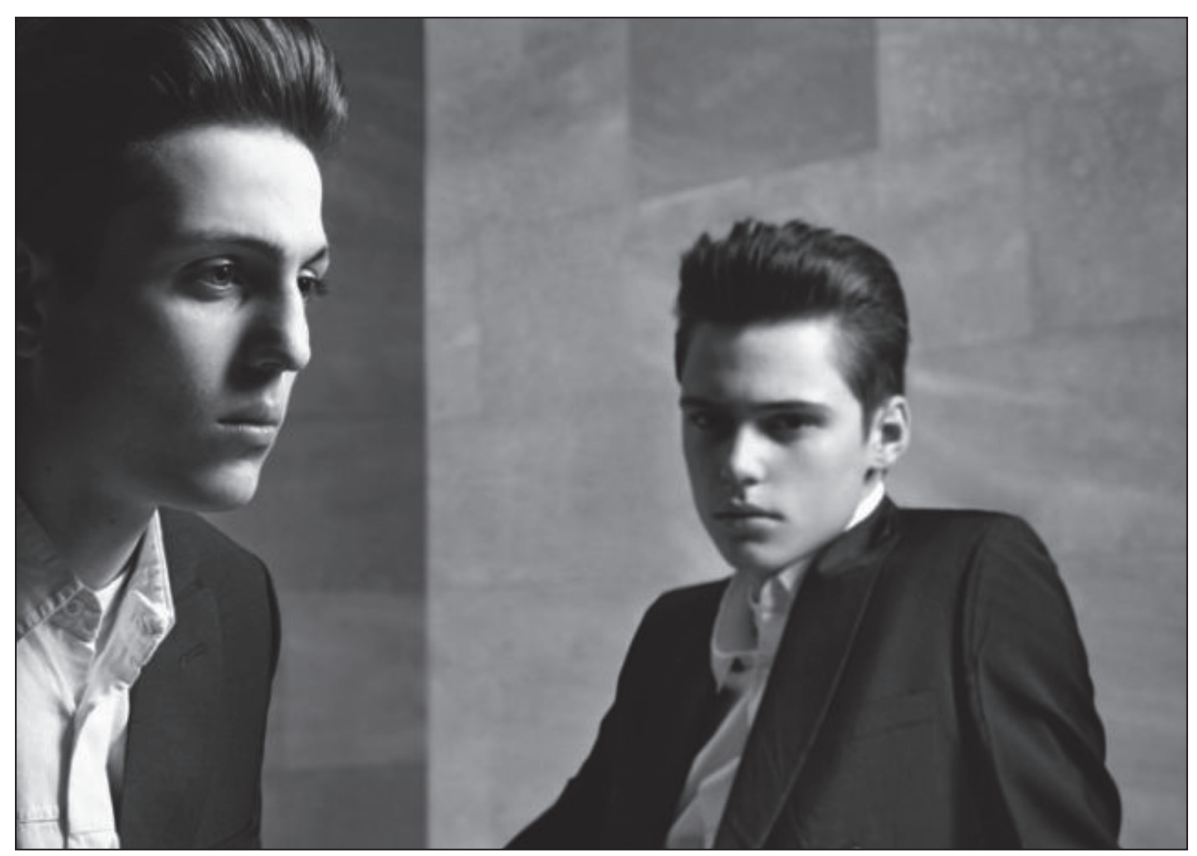

Immagine 2. - Prada Primavera-Estate 2009.

Fotografo: Hedi Slimane - Stylist: Olivier Rizzo. 


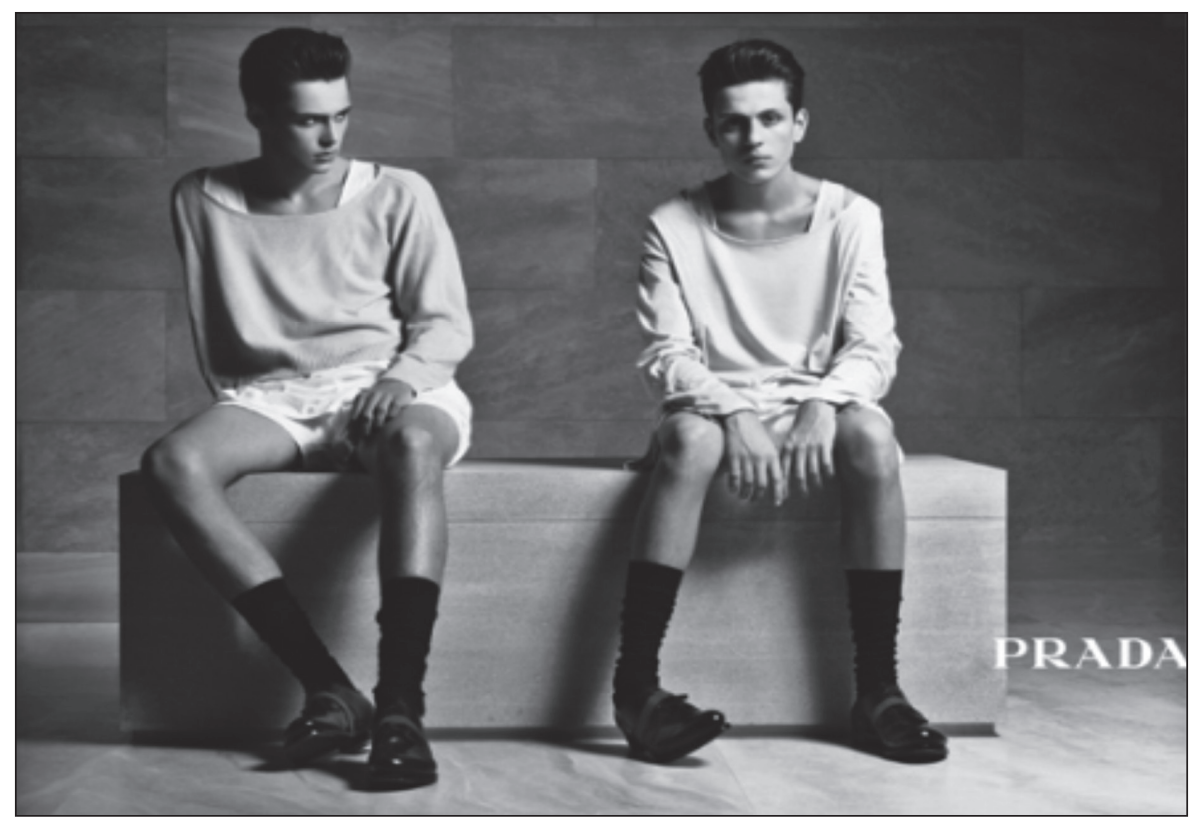

Immagine 3. - Prada Primavera-Estate 2009.

Fotografo: Hedi Slimane - Stylist: Olivier Rizzo.

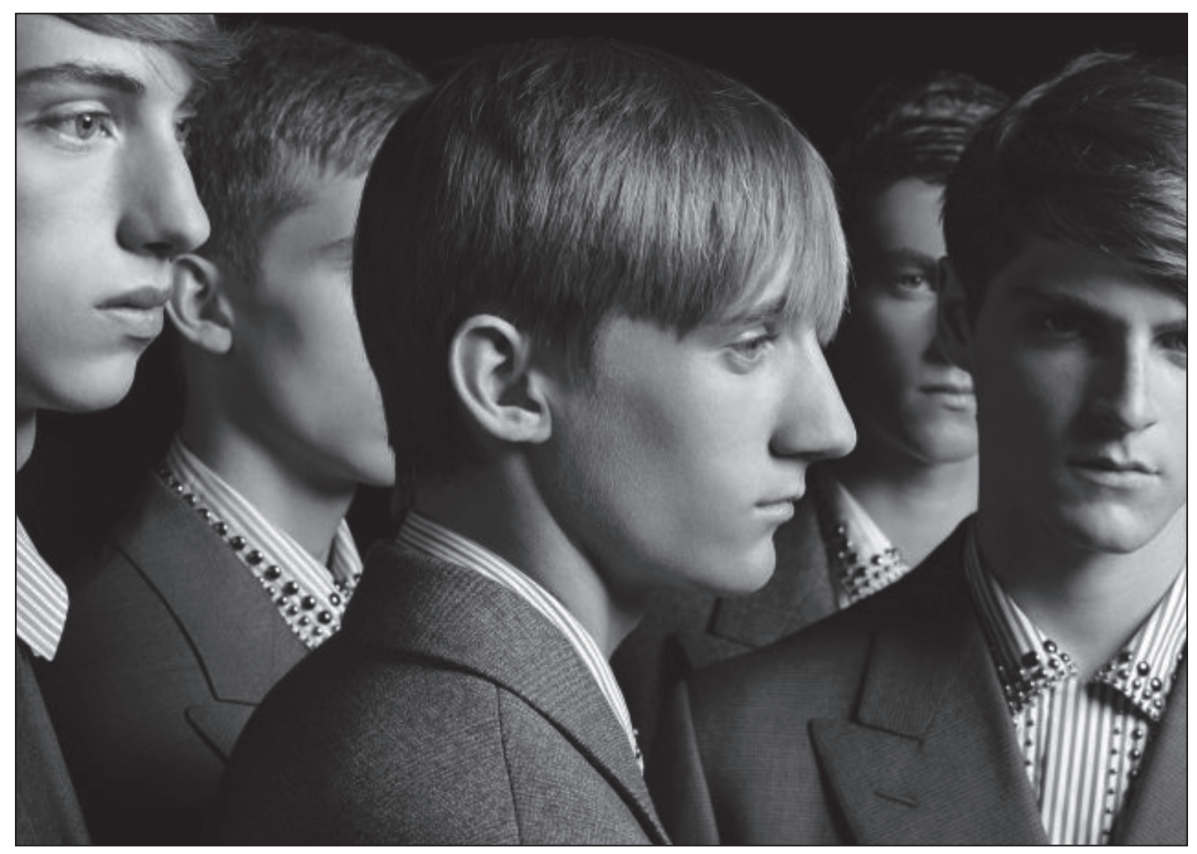

Immagine 4. - Prada Autunno-Inverno 2009.

Fotografo: Hedi Slimane - Stylist: Olivier Rizzo. 


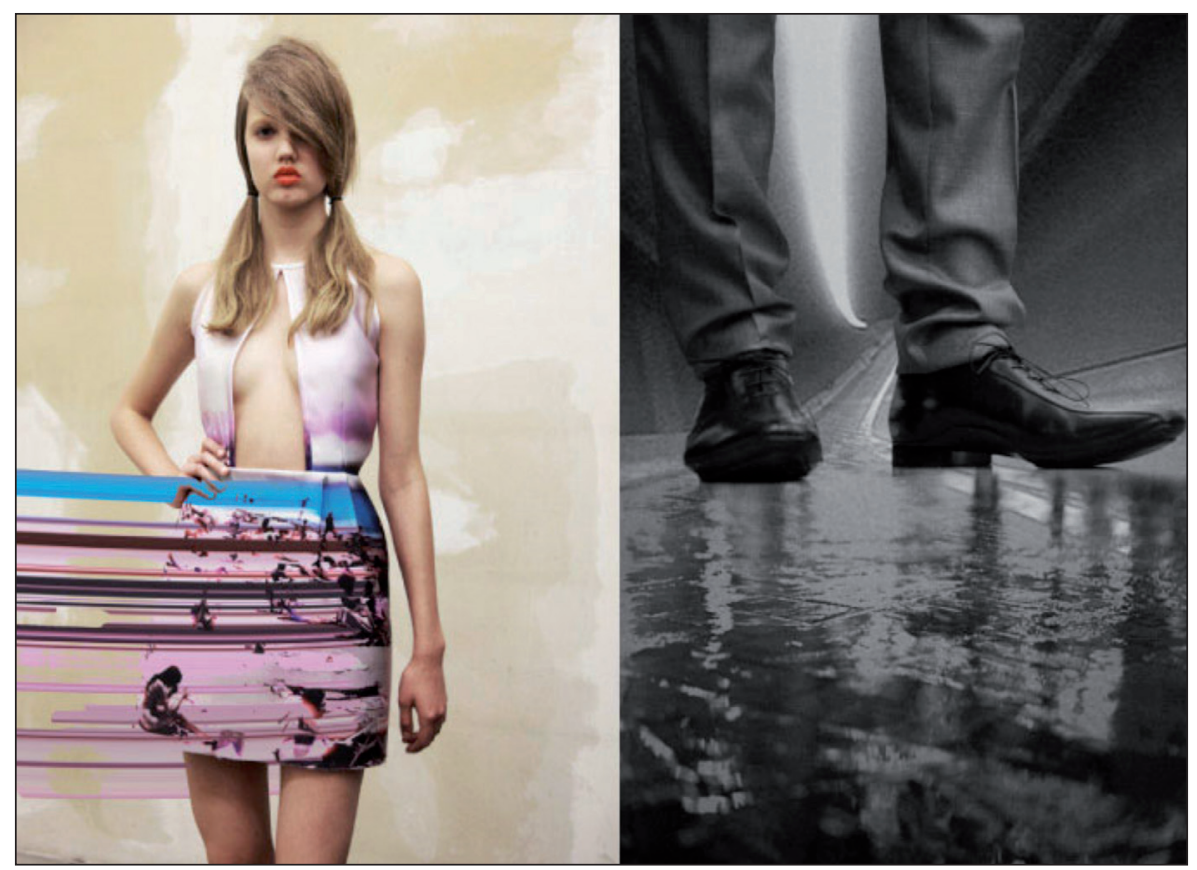

Immagine 5.

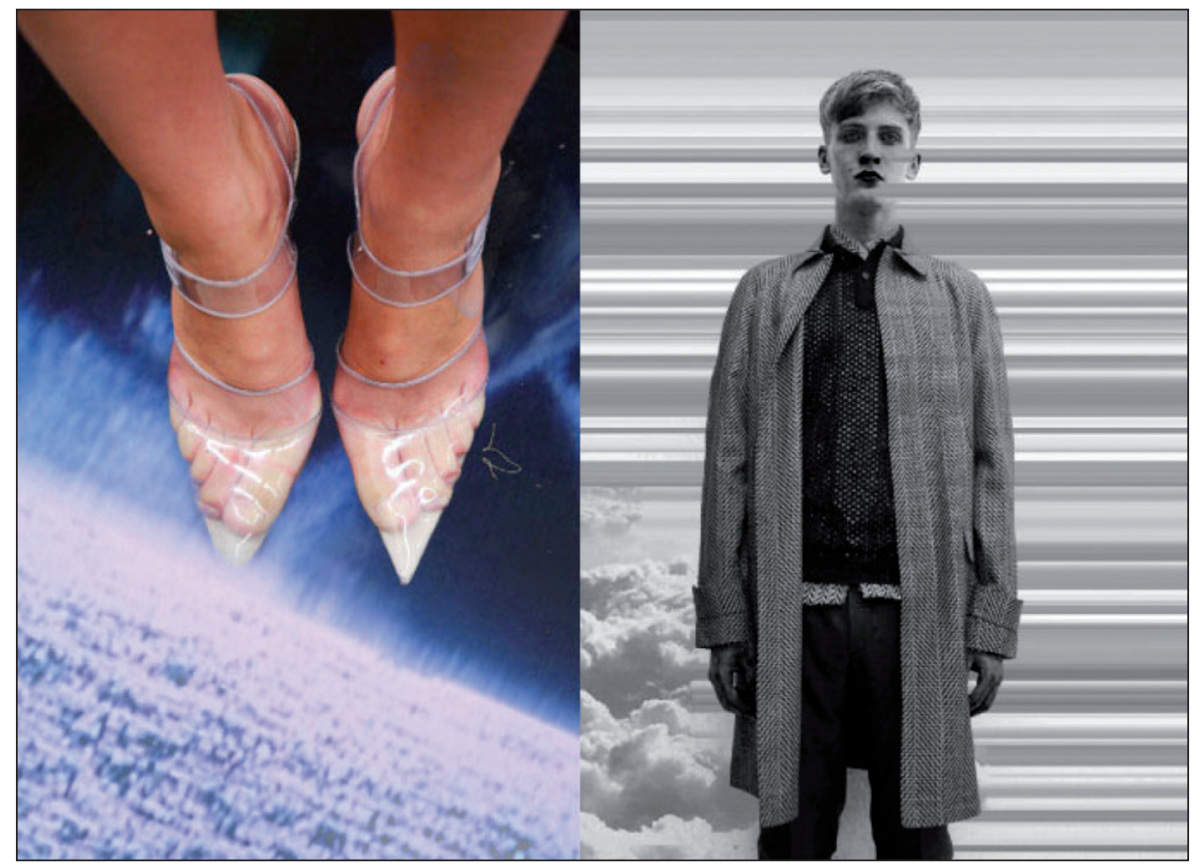

Immagine 6. 


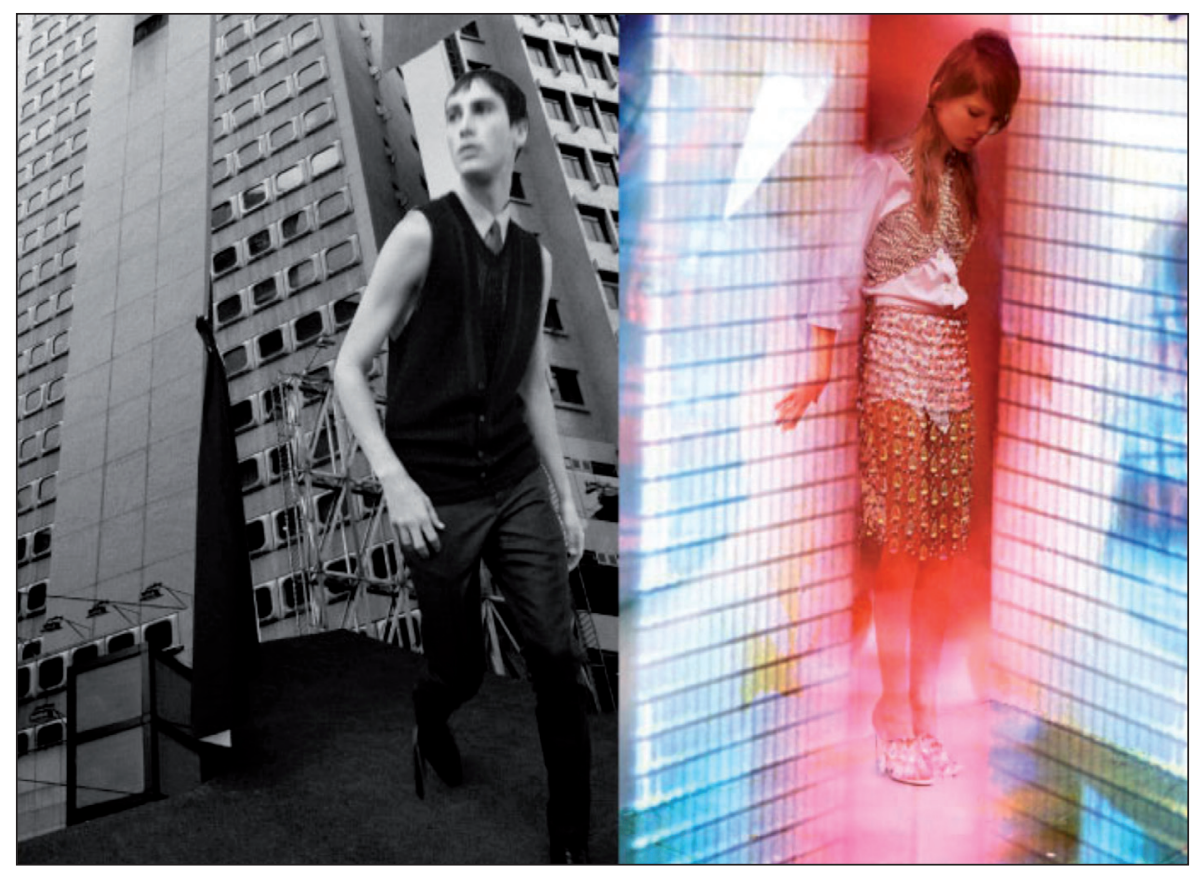

Immagine 7. - Prada Primavera-Estate 2010.

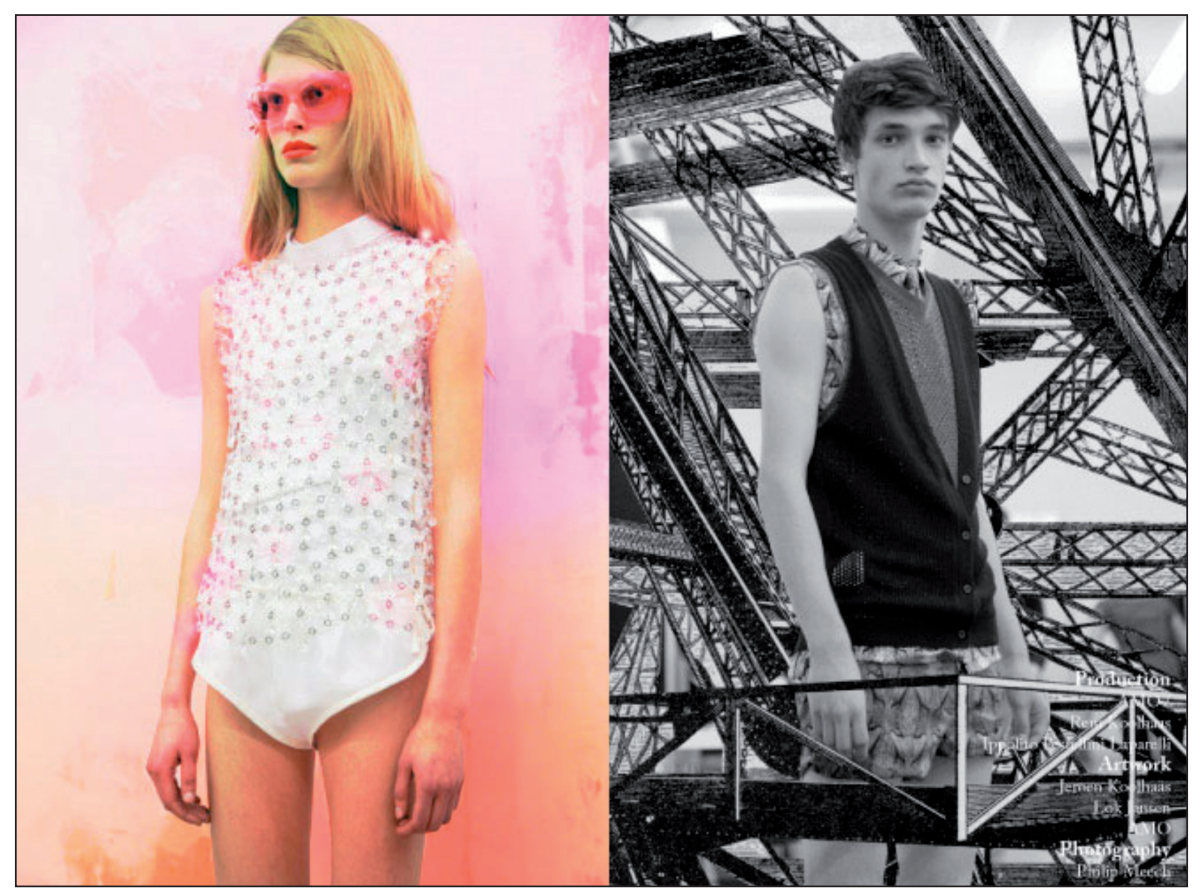

Immagine 8 . 


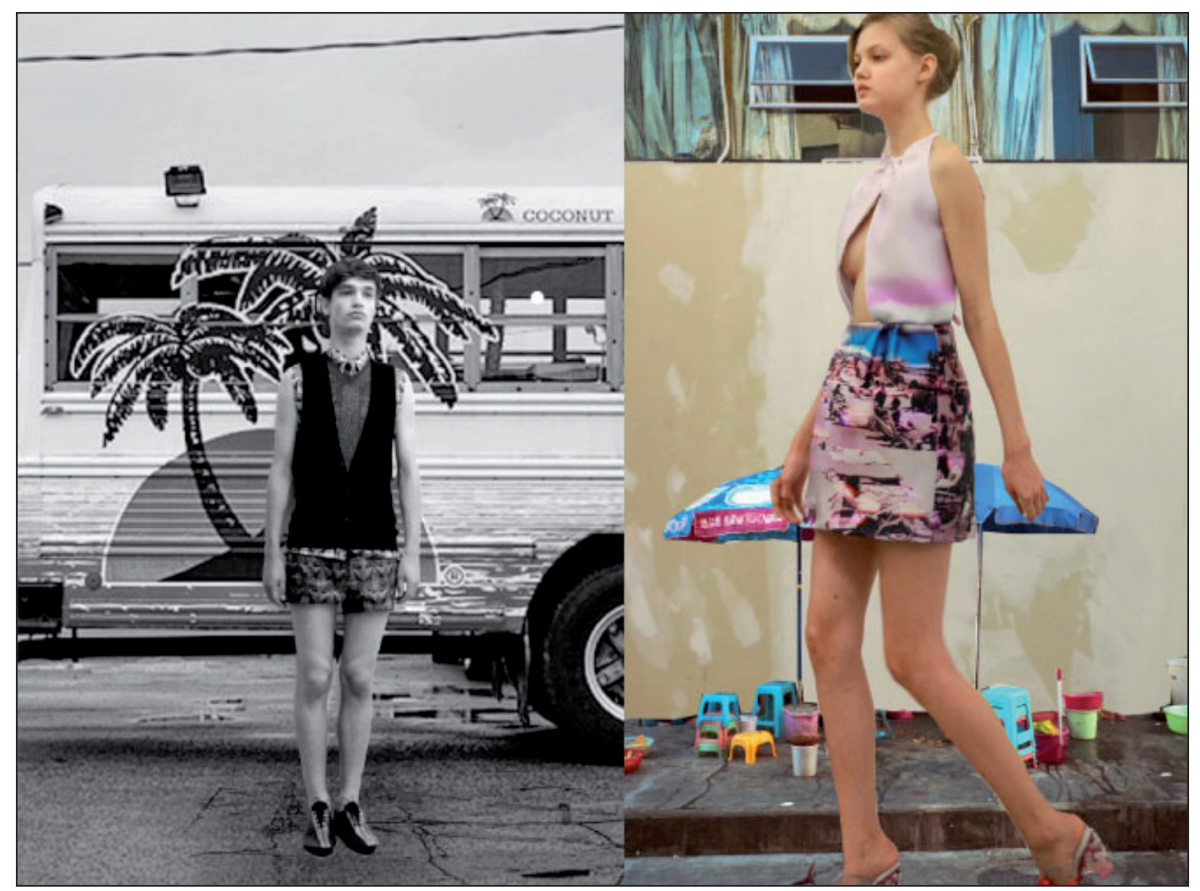

Immagine 9.

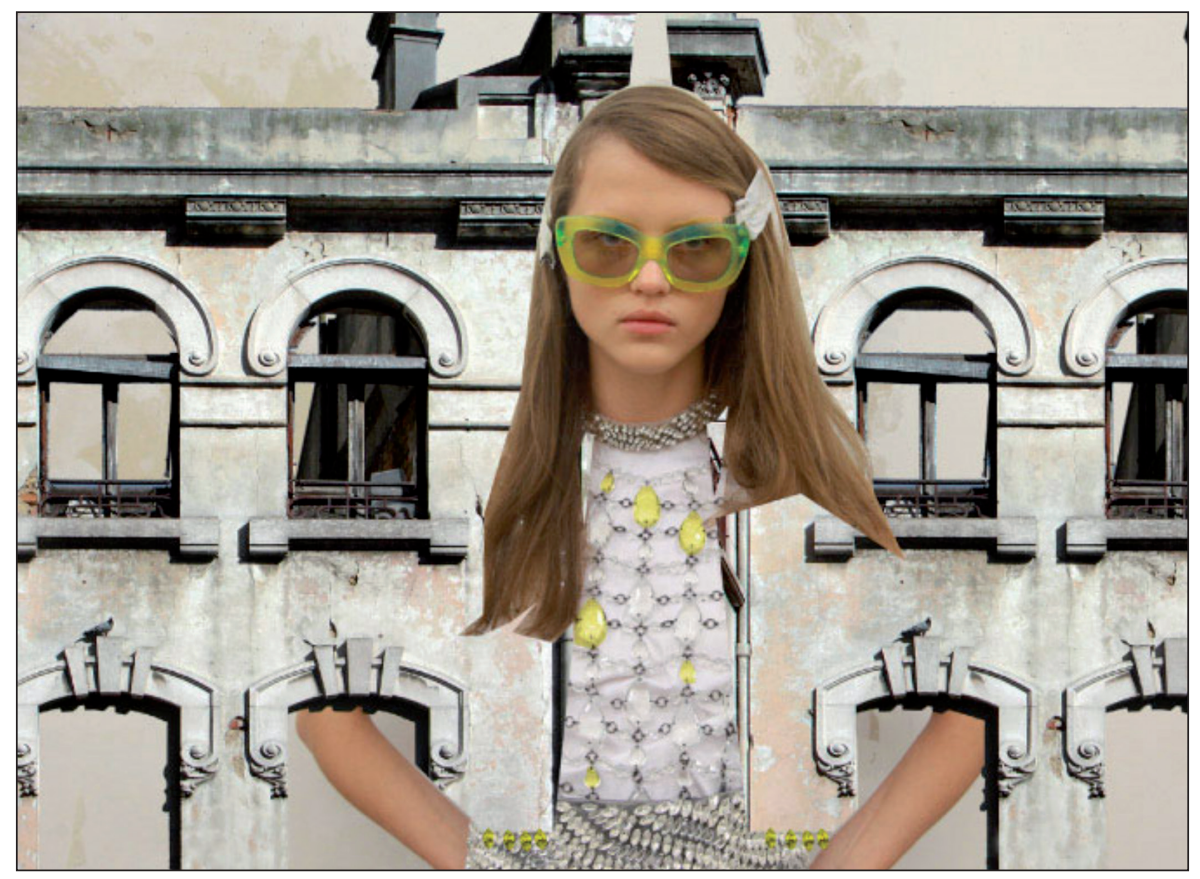

Immagine IO. 


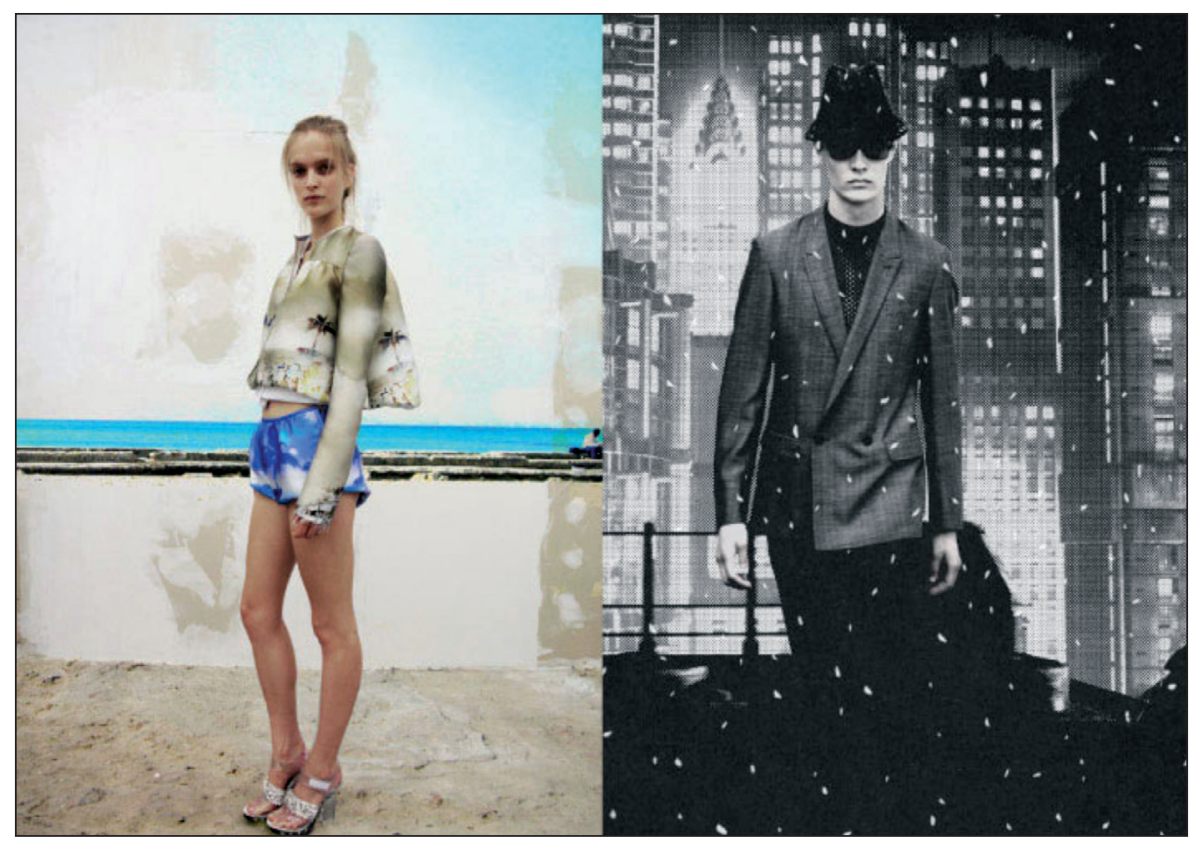

Immagine II.

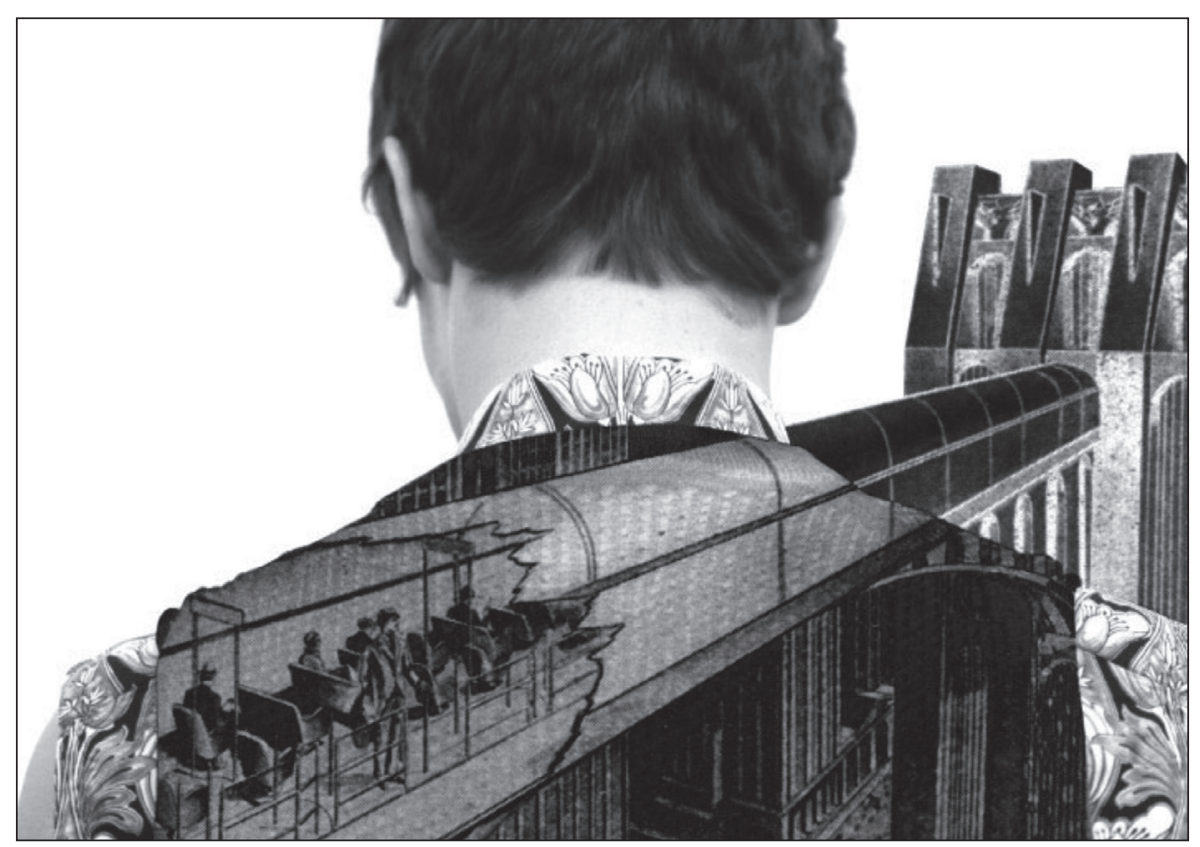

Immagine I2. 